\title{
Vitamina D, obesidad y COVID-19 en pediatría
}

\author{
Vitamin D, obesity and COVID-19 in pediatrics
}

\author{
Jessie Nallely Zurita-Cruz,* Sergio Alberto Pérez-Cervantes ${ }^{\ddagger}$ \\ * Facultad de Medicina, Universidad Nacional Autónoma de México, Hospital Infantil de México \\ Federico Gómez; ${ }^{\ddagger}$ Residente. Servicio de Endocrinología Pediátrica, UMAE Hospital de Pediatría, Centro \\ Médico Nacional Siglo XXI, Instituto Mexicano del Seguro Social, Ciudad de México.
}

La vitamina $\mathrm{D}$ es fundamental en la homeostasis del calcio y el fósforo y, en particular, de las vías involucradas en la mineralización ósea y de la adquisición de masa ósea. Además de estas acciones esqueléticas, que se conocen desde hace muchos años, estudios recientes han demostrado que puede tener algún papel en la patogénesis de enfermedades infecciosas y autoinmunes. ${ }^{1}$

El término vitamina $\mathrm{D}(\mathrm{VD})$ se usa para identificar las dos formas que se encuentran en la naturaleza: VD3 (colecalciferol) de origen animal y VD2 (ergocalciferol) de plantas. Los humanos sintetizan la VD3 en la piel, en respuesta a la exposición a la luz solar, pero también puede ser adquirida por fuentes dietéticas, lo mismo que la VD2. Aunque existe en muchos alimentos, sólo algunos contienen cantidades significativas, como la leche, yogurt, queso, pescados (sardinas, arenques, atún, caballa, salmón, aceite de hígado de bacalao), yemas de huevo, hígado, hongo shiitake y vísceras. ${ }^{2-4}$

Alrededor de $90 \%$ de la VD proviene de la síntesis cutánea a través de la conversión de 7-dehidrocolesterol a precolecalciferol por la luz ultravioleta del sol. El precolecalciferol es una molécula muy inestable que rápidamente se convierte en colecalciferol. Es necesario agregar dos hidroxilaciones para que el colecalciferol se trasforme en la molécula activa. La primera hidroxilación se realiza a nivel hepático por la 25-hidroxilasa que forma la $25(\mathrm{OH}) \mathrm{D}$, y después se forma la 1-25(OH) D (calcitriol) a nivel renal por la 1-alfa hidroxilasa. ${ }^{5}$
La producción cutánea de VD3 está influenciada por varios factores, como la pigmentación de la piel, el porcentaje de área de piel expuesta, el tiempo de exposición diaria al sol, el tipo de ropa y el uso de protector solar. Asimismo, participan factores ambientales, como la latitud, altitud, estaciones del año y la contaminación atmosférica. En comparación a los adultos, los niños requieren menos exposición a la luz para producir cantidades suficientes de VD, tanto por su mayor proporción de superficie corporal, como porque tienen mejor capacidad para producir VD. ${ }^{6}$

La concentración sérica de $25(\mathrm{OH}) \mathrm{D}$ se considera el marcador más preciso para determinar el estado de VD en el cuerpo. ${ }^{7}$ La Sociedad Internacional de Endocrinología propone que los niveles por debajo de $20 \mathrm{ng} / \mathrm{mL}(50 \mathrm{nmol} / \mathrm{L})$ corresponden a deficiencia, los niveles que van de 20 a $29.9 \mathrm{ng} / \mathrm{mL}(52-72 \mathrm{nmol} / \mathrm{L})$ a insuficiencia y, $>30 \mathrm{ng} / \mathrm{mL}$ ( $75 \mathrm{nmol} / \mathrm{L}$ ) identifican los niveles normales o la suficiencia. Esta clasificación se basa, en gran medida, en los efectos de la VD sobre la homeostasis ósea y mineral. Sin embargo, el nivel sérico óptimo de 25(OH)D con respecto a otros aspectos de la salud humana aún no se ha descrito. ${ }^{8}$ Existen condiciones o factores que modifican las concentraciones séricas de VD, entre las que encuentran la mala absorción intestinal, enfermedades crónicas y obesidad. ${ }^{9}$

Los expertos sugieren que para producir efectos benéficos en enfermedades relacionadas con el sistema

Correspondencia: Jessie Nallely Zurita-Cruz, E-mail: zuritajn@hotmail.com

Citar como: Zurita-Cruz JN, Pérez-Cervantes SA. Vitamina D, obesidad y COVID-19 en pediatría. Rev Mex Pediatr. 2021; 88(4): 129-132. https://dx.doi.org/10.35366/102776 
inmunológico se pueden necesitar niveles séricos de 25(OH)D mayores, $40-60 \mathrm{ng} / \mathrm{mL}(100-150 \mathrm{nmol} / \mathrm{L}) .{ }^{10}$

De acuerdo con las recomendaciones internacionales, la suplementación de VD en población pediátrica se modifica por la edad, particularmente desde recién nacido hasta los 12 meses. Así, los niños sin factores de riesgo (de deficiencia de VD) deben recibir 400 UI/ día, mientras que en presencia de factores de riesgo se pueden administrar hasta 1,000 UI/día. En el caso de niños de uno a 18 años, el aporte de VD recomendado varía de $400 \mathrm{UI} /$ día hasta 1,000 UI/día. ${ }^{1,6}$ En este mismo grupo, cuando se detecta deficiencia de VD se recomienda 2,000 UI/día durante seis semanas, hasta alcanzar niveles de 25 a $30 \mathrm{ng} / \mathrm{mL}$; posteriormente hasta 1,000 UI/día. ${ }^{1}$

\section{OBESIDAD EN NIÑOS Y VITAMINA D}

Desde hace algunas décadas, la obesidad infantil ha sido un problema en México, el cual, al parecer, se aceleró durante la pandemia por COVID-19, debido al aislamiento social. ${ }^{11}$ Se conoce que los pacientes con obesidad tienen niveles bajo de VD. Sin embargo, no existe un consenso sobre las causas relacionadas a que los niveles de calcitriol sean bajos. La teoría más aceptada es que el tejido adiposo absorbe la VD liposoluble. Otra explicación asocia el sedentarismo y la baja exposición a la luz solar, con una disminución en la síntesis endógena de VD. Igualmente se ha propuesto que la esteatosis hepática, desarrollada en el marco de un paciente con obesidad, disminuye la síntesis de $25(\mathrm{OH}) \mathrm{D}$ por la afección en el metabolismo hepático. Por último, también se ha señalado que niveles altos de leptina e IL-6 impiden la síntesis de $25(\mathrm{OH}) \mathrm{D}$, afectando los receptores de VD. ${ }^{12}$

\section{VITAMINA D EN LA PREVENCIÓN DE INFECCIONES}

Respecto al papel preventivo de la VD en las infecciones respiratorias agudas, se han identificado algunos aspectos fisiopatológicos. Las células presentadoras de antígenos innatas son responsables del inicio de la respuesta inmune adaptativa, ya que presentan antígenos para las células T y B y son capaces de modularlos mediante señales inmunogénicas, como las citocinas y la expresión de moléculas coestimuladoras. ${ }^{13}$ Existen estudios donde se demuestra que el calcitriol y sus análogos pueden alterar la función y la morfología de células dendríticas, induciendo un estado inmaduro más tolerogénico. ${ }^{14-16}$ También se ha descrito que el calcitriol inhibe las citocinas de las células T, como la IL-2 y la IL-17, produciendo receptores similares en los monocitos. ${ }^{17}$

La VD mejora la inmunidad innata celular, en parte, a través de la inducción de péptidos antimicrobianos, incluida la catelicidina humana, LL-37, por 1,25-dihdroxivitamina D y defensinas en el epitelio respiratorio. ${ }^{18-20}$ Las catelicidinas exhiben actividades antimicrobianas directas contra un espectro de microbios, incluyendo bacterias Gram-positivas y Gramnegativas, virus y hongos envueltos y no envueltos. ${ }^{21}$ Esos péptidos derivados del huésped eliminan patógenos invasores al perturbar sus membranas celulares, y también pueden neutralizar las actividades biológicas de las endotoxinas. ${ }^{22,23}$

En estudios observacionales prospectivos, se ha comprobado el efecto protector de la VD en niños sanos de tres a 15 años. Al comparar niños con una concentración de $25(\mathrm{OH}) \mathrm{D}$ de $20 \mathrm{ng} / \mathrm{mL}$ (75 nmol/L), y aquéllos con $25(\mathrm{OH}) \mathrm{D}<20 \mathrm{ng} / \mathrm{mL}(75 \mathrm{nmol} / \mathrm{L})$, se demostró mayor incidencia de infecciones gastrointestinales y del oído (HR 2.05; IC 95\% 1.19-3.53). Mientras que quienes tenían $<20 \mathrm{ng} / \mathrm{mL}(50 \mathrm{nmol} / \mathrm{L})$ de $25(\mathrm{OH}) \mathrm{D}$ tuvieron el mayor riesgo de desarrollar infección viral de vías aéreas (HR, 1.67; IC 95\%, 1.16-2.40, p = 0.006). ${ }^{24,25}$

Basado en estudios como los señalados en el párrafo previo, se han realizado ensayos clínicos aleatorizados (ECA) para evaluar el efecto de la suplementación con VD en la prevención de infecciones del tracto respiratorio en niños. ${ }^{26,27}$ Sin embargo, hasta el momento no es claro si la VD produce algún efecto protector en población pediátrica, ya que en estos ECA tienen problemas metodológicos, como tamaño de muestra pequeños, características diversas de los participantes, así como diferentes dosis y tiempos de administración de VD. En un metaanálisis publicado en 2015, donde se incluyeron siete ECA, no se identificó asociación entre la suplementación de VD y la reducción en el riesgo de presentar infecciones respiratorias agudas en población pediátrica. ${ }^{28}$

En contraste, recientemente se publicó otro metaanálisis con el objetivo de determinar el efecto de la suplementación con VD sobre el riesgo de infecciones respiratorias agudas, así como el régimen de dosificación. Se incluyeron 46 ECA (49,419 participantes desde recién nacidos hasta pacientes de 95 años). Al comparar los resultados entre la suplementación con VD y placebo, se determinó que la VD redujo el riesgo de infecciones respiratorias agudas (Odds Ratio [OR] 0.91; IC95\% 0.84-0.99). Los efectos protectores se observaron en los ECA en los que se administró VD 
en forma diaria (OR 0.75; IC95\% 0.61 a 0.93), a dosis diarias de 400 a 1,000 UI (OR 0.70; IC95\% 0.55 a 0.89 ), y por una duración $\leq 12$ meses (OR 0.82; IC95\% 0.72 a 0.93). Es importante señalar que las concentraciones iniciales de $25(\mathrm{OH}) \mathrm{D}$ no tuvieron impacto sobre el riesgo de presentar infecciones respiratorias agudas. Los autores concluyen que la suplementación con VD parece reducir el riesgo de infecciones respiratorias agudas, pero debido a que los estudios son muy distintos, todavía no es posible recomendar de manera definitiva la suplementación de VD a nivel poblacional, incluyendo a los niños. ${ }^{29}$

\section{VITAMINA D Y COVID-19}

La enfermedad por coronavirus 2019 (COVID-19) se asocia con una mayor producción de citocinas proinflamatorias, ${ }^{30}$ proteína $\mathrm{C}$ reactiva, mayor riesgo de neumonía, sepsis, síndrome de dificultad respiratoria aguda e insuficiencia cardiaca. ${ }^{31} \mathrm{~A}$ lo largo de la pandemia, se ha observado que los niños constituyen de 1-5\% de los casos diagnosticados con COVID-19, pero en comparación a pacientes adultos, en general, cursan con enfermedad leve. ${ }^{32}$ Sin embargo, se ha puesto en evidencia que niños menores de tres años de edad y con alguna comorbilidad (especialmente diabetes, obesidad y con problemas de inmunidad) presentan mayor riesgo de desarrollar enfermedad grave. ${ }^{33}$

En un metaanálisis de estudios observacionales publicado recientemente sobre la relación entre el nivel de VD, el riesgo y la gravedad de COVID-19 en pediatría, se demostró una prevalencia de deficiencia de VD de 45.9\% en pacientes pediátricos con COVID-19. Encontrando, además, que los niños y adolescentes que tenían deficiencia de VD tenían un mayor riesgo de infección por COVID-19, en comparación con los pacientes con niveles normales de VD. Mientras que en los pacientes infectados, los niveles bajos de VD aumentaron el riesgo de enfermedad grave (OR 5.5; IC95\% 1.5-19.5; $\mathrm{p}=0.008) .{ }^{34}$

Como consecuencia de los datos previamente descritos, desde el inicio de la pandemia se ha planteado que la suplementación con VD podría ser benéfica y se han propuesto ECA; sin embargo, la efectividad de la suplementación de VD en COVID-19 todavía no está disponible porque aún no hay publicaciones que lo avalen. Es probable que pronto tengamos resultados, ya que al menos nueve ECA se han registrado en la plataforma de Clinical Trials sobre el efecto de la suplementación con VD para prevenir COVID-19 o disminuir el riesgo de una evolución grave. ${ }^{35}$
Como conclusión podemos afirmar que, aunque la evidencia en pediatría es limitada, dado el escenario que actualmente estamos viendo en esta época de pandemia, podría ser apropiado que en todo caso con sospecha de COVID-19 y que requiera realizar estudios de laboratorio, incluir la determinación de los niveles séricos de $25(\mathrm{OH}) \mathrm{D}$. En casos con deficiencia o insuficiencia, la suplementación de VD parece estar justificada.

\section{REFERENCIAS}

1. Saggese G, Vierucci F, Boot AM, Czech-Kowalska J, Weber G, Camargo CA Jr. et al. Vitamin D in childhood and adolescence: an expert position statement. Eur J Pediatr. 2015; 174 (5): 565-576.

2. Chang SW, Lee HC. Vitamin D and health - The missing vitamin in humans. Pediatr Neonatol. 2019; 60 (3): 237-244.

3. Elizondo-Montemayor L, Ugalde-casas PA, SerranoGonzález M, Cuello-García CA, Borbolla-Escoboza JR. Serum 25-hydroxyvitamin D concentration, life factors and obesity in Mexican children. Obesity (Silver Spring). 2009; 18 (9): 1805-1811.

4. Hossein-Nezhad A, Holick MF. Vitamin D for health: a global perspective. Mayo Clin Proc. 2013; 88 (7): 720-755.

5. Gröber U, Spitz J, Reichrath J, Kisters K, Holick MF. Vitamin D: update 2013: from rickets prophylaxis to general preventive healthcare. Dermatoendocrinol. 2013; 5 (3): 331-347.

6. Hossein-nezhad A, Holick MF. Vitamin D for health: a global perspective. Mayo Clin Proc. 2013; 88 (7): 720-755.

7. Holick MF. Vitamin D deficiency. N Engl J Med. 2007; 357: 266281.

8. Holick MF, Binkley NC, Bischoff-Ferrari HA, Gordon CM, Hanley DA, Heaney RP et al. Evaluation, treatment, and prevention of vitamin $\mathrm{D}$ deficiency: an Endocrine Society clinical practice guideline. JCEM. 2011; 96: 1911-1930.

9. Wang $\mathrm{H}$, Chen W, Li D, Yin X, Zhang X, Olsen $\mathrm{N}$ et al. Vitamin $\mathrm{D}$ and chronic diseases. Aging Dis. 2017; 8 (3): 346-353.

10. Hewison, M. An update on vitamin $D$ and human immunity. Clin Endocrinol (Oxt). 2012; 76 (3): 315-325.

11. Nogueira-de-almeida CA, Del LA, Ferraz IS, Del IRL, Contini AA, Ued V. COVID-19 and obesity in childhood and adolescence: a clinical review. J Pediatr (Rio J). 2020; 96 (5): 546-558.

12. Zakharova I, Klimov L, Kuryaninova V, Nikitina I, Malyavskaya S, Dolbnya $S$ et al. Vitamin D insufficiency in overweight and obese children and adolescents. Front Endocrinol (Lausanne). 2019; 10: 103.

13. Holick MF. Sunlight and vitamin D for bone health and prevention of autoimmune disease, cancers and cardiovascular disease. Am $J$ Clin Nutr. 2004; 80 (60 Suppl): 678S-688S.

14. Steinman RM, Banchereau J. Taking dendritic cells into medicine. Nature. 2007; 449 (7161): 419-426.

15. Penna G, Amuchastegui S, Giarratana N, Daniel KC, Vulcano M, Sozzani S. 1,25-Dihydroxyvitamin D3 selectively modulates tolerogenic properties in myeloid but not plasmacytoid dendritic cells. J Immunol. 2007; 178 (1): 145-153.

16. Veldman CM, Cantorna MT, DeLuca HF. Expression of 1,25-dihydroxyvitamin D3 receptor in the immune system. Arch Biochem Biophys. 2000; 374 (2): 334-338.

17. Banchereau J, Steinman RM. Dendritic cells and the control of immunity. Nature. 1998; 392 (6673): 245-252.

18. Wang TT, Nestel FP, Bourdeau V, Nagai $Y$, Wang $Q$, Liao J et al. Cutting edge: 1,25-dihydroxyvitamin D3 is a direct inducer of 
antimicrobial peptide gene expression. J Immunol. 2004; 173 (5): 2909-2912.

19. Laaksi I. Vitamin $D$ and respiratory infection in adults. Proc Nutr Soc. 2012; 71: 90-97.

20. Herr C, Shaykhiev R, Bals R. The role of cathelicidin and defensins in pulmonary inflammatory diseases. Expert Opin Biol Ther. 2007; 7 (9): 1449-1461.

21. Agier J, Efenberger M, Brzezinska-Blaszczyk E. Cathelicidin impact on inflammatory cells. Cent Eur J Immunol. 2015; 40 (2): 225-235.

22. Barlow PG, Svoboda P, Mackellar A, Nash AA, York IA, Pohl J et al. Antiviral activity and increased host defense against influenza infection elicited by the human cathelicidin LL-37. PLoS One. 2011; 6 (10): e25333.

23. Adams JS, Ren S, Liu PT, Chun RF, Lagishetty V, Gombart AF et al. Vitamin d-directed rheostatic regulation of monocyte antibacterial responses. J Immunol. 2009; 182 (7): 4289-4295.

24. Thornton KA, Marin C, Mora-Plazas M, Villamor E. Vitamin D deficiency associated with increased incidence of gastrointestinal and ear infections in school-age children. Pediatr Infect Dis J. 2013; 32 (6): 585-593.

25. Science M, Maguire JL, Russell ML, Smieja M, Walter SD, Loeb M. Low serum 25-hydroxyvitamin D level and risk of upper respiratory tract infection in children and adolescents. Clin Infect Dis. 2013; 57 (3): 392-397.

26. Urashima M, Segawa T, Okazaki M, KuriharaM, Wada Y, Ida H. Randomized trial of vitamin D supplementation to prevent seasonal influenza: a in schoolchildren. Am J Clin Nutr. 2010; 91 (5): 12551260.

27. Grant CC, Kaur S,Waymouth E, Mitchell EA, Scragg R, Ekeroma A et al. Reduced primary care respiratory infection visits following pregnancy and infancy vitamin $D$ supplementation: a randomised controlled trial. Acta Paediatr. 2015; 104 (4): 396-404.

28. Xiao L, Xing C, Yang Z, Xu S, Wang M, Du H et al. Vitamin D supplementation for the prevention of childhood acute respiratory infections: a systematic review of randomised controlled trials. $\mathrm{Br}$ J Nutr. 2015; 114 (7): 1026-1034.

29. Jolliffe DA, Camargo CA Jr, Sluyter JD, Aglipay M, Aloia JF, Ganmaa D et al. Vitamin D supplementation to prevent acute respiratory infections: systematic review and meta-analysis of aggregate data from randomised controlled trials. MedRxiv [Preprint]. 2020: 2020.07.14.20152728.

30. Wang D, Hu B, Hu C, Zhu F, Liu X, Zhang J et al. Clinical characteristics of 138 hospitalized patients with 2019 novel coronavirus-infected pneumonia in Wuhan, China. JAMA. 2020; 323 (11): 1061-1069.

31. Zhou F, Yu T, Du R, Fan G, Liu Y, Liu Z et al. Clinical course and risk factors for mortality of adult inpatients with COVID-19 in Wuhan, China: A retrospective cohort study. Lancet. 2020; 395 (10229): 1054-1062.

32. Ludvigsson JF. Systematic review of COVID-19 in children show milder cases and a better prognosis than adults. Acta Paediatr. 2020; 109 (6): 1088-1095.

33. Moreno-Noguez M, Rivas-Ruiz R, Roy-García IA, PachecoRosas DO, Moreno-Espinosa S, Flores-Pulido AA. Factores de riesgo asociados con neumonía por SARS-CoV-2 en población pediatrica. Bol Med Hosp Infant Mex. 2021; 78 (4): 251-258.

34. Shah K, Varna VP, Pandya A, Saxena D. Low vitamin D levels and prognosis in a COVID-19 pediatric population: a systematic review. QJM. 2021; 114 (7): 447-453.

35. U.S. National Library of Medicine [Internet]. ClinicalTrials.gov. 2021 [29 November 2021]. Available in: https://clinicaltrials.gov/ct2/result s?term=vitamin+D\&cond=COVID-19+Respiratory+Infection\&age_ $\mathrm{V}=\& g n d r=\&$ type $=$ Intr\&rsIt=\&Search=Apply

Conflicto de intereses: los autores declaran que no tienen. 